\title{
Atenção Primária e Coordenação do Cuidado: dispositivo para ampliação do acesso e a melhoria da qualidade
}

\author{
Primary Health Care and Coordination of Care: device to increase \\ access and improve quality
}

Sabiny Pedreira Ribeiro (https://orcid.org/0000-0001-7151-4605) ${ }^{1}$

Maria de Lourdes Tavares Cavalcanti (https://orcid.org/0000-0002-4929-1123) ${ }^{1}$

${ }^{1}$ Instituto de Estudos em Saúde Coletiva, Universidade Federal do Rio de Janeiro. Av. Hirácio Macedo s/n, Ilha do Fundão, Cidade Universitária. Rio de Janeiro RJ Brasil. sabiny.ribeiro@gmail.com

\begin{abstract}
The objective of this article is to investigate the viewpoint of health professionals regarding the coordination of care in Primary Health Care (PHC) and the challenges for performing it. Qualitative research with professionals from two PHC teams working in territories with vulnerabilities and inequalities in Rio de Janeiro. Use of the Content Analysis method, thematic modality with semi-structured interviews and participant observation. To coordinate care is to be involved with the activities that foster the provision of individualized and comprehensive care, aiming at the care continuity. The challenges of coordinating care by the PHC are overcoming the network fragmentation, the low supply of openings for specialists, the fragile communication between the services, the non-integrated electronic medical record, the low professional qualification and the unawareness of the PHC role by other services. The strengthening of the PHC as the gateway, coordinator and organizer of the network requires efforts by managers, professionals and society, so this way of organizing the systems can bring benefits regarding equity, accessibility, clinical and sanitary effectiveness, economic efficiency and allow care integration.
\end{abstract}

Key words Primary Health Care, Health services, Comprehensive health care
Resumo O objetivo deste artigo é investigar a visão dos profissionais de saúde a respeito da coordenação do cuidado na Atenção Primária à Saúde (APS) e os desafios frente ao seu exercício. Pesquisa qualitativa com profissionais de duas equipes de APS que atuam em territórios com vulnerabilidades e desigualdades no Rio de Janeiro. Utilização do método de Análise de Conteúdo, modalidade temática com realização de entrevistas semiestruturadas e observação participante. Coordenar o cuidado é estar envolvido com as atividades que proporcionam a prestação de um cuidado individualizado e integral, visando a continuidade do cuidado. Os desafios da coordenação do cuidado pela APS são a superação da fragmentação da rede, a baixa oferta de vagas para especialistas, a frágil comunicação entre os serviços, o prontuário eletrônico não integrado, a baixa qualificação profissional e o desconhecimento do papel da APS pelos outros serviços. O fortalecimento da APS como porta de entrada, coordenadora e ordenadora da rede requer esforços de gestores, profissionais e sociedade, a fim de que esse modo de organização dos sistemas possa trazer benefícios no que tange à equidade, à acessibilidade, à eficácia clínica e sanitária, à eficiência econômica e que possibilite a integração dos cuidados.

Palavras-chave Atenção Primária à Saúde, Serviços de saúde, Assistência integral à saúde 


\section{Introdução}

A fragmentação do cuidado à saúde ${ }^{1,2}$ aliada à mudanças demográficas e epidemiológicas e agravada pelas desigualdades no acesso aos serviços, constitui um desafio aos sistemas de saúde. Pessoas portadoras de doenças crônicas, com mais fragilidades e incapacidades, bem como aquelas em situação de maior vulnerabilidade social, necessitam de mais cuidados e demandam serviços com maior frequência e concomitantes em diferentes especialidades e níveis de atenção, resultando em interdependência entre os pontos da rede de atenção $0^{3-6}$. A fragmentação sistêmica traz consequências negativas à eficiência e efetividade das ações em saúde, contribuindo para $o$ aumento das iniquidades e vulnerabilidade no que tange, sobretudo, ao acesso e utilização dos serviços e integralidade do cuidado ${ }^{7,8}$.

A integração em redes de atenção à saúde (RAS) opõe-se à fragmentação, promove integração sistêmica provendo atenção contínua, integral, de qualidade, responsável e humanizada, e incrementa o desempenho do sistema em termos de acesso, equidade, eficácia clínica e sanitária e eficiência econômica ${ }^{9-14}$.

A Atenção Primária à Saúde (APS) é considerada a principal estratégia de reorientação do modelo assistencial ${ }^{15}$, apontada como centro de comunicação das redes e nó intercambiador coordenador de fluxos e contrafluxos ${ }^{11,12}$. Uma APS como porta de entrada, não focalizada em grupos em situação de pobreza, capaz de assumir a coordenação do cuidado dos usuários ${ }^{16,17}$ como um atributo fundamental na organização de sistemas de saúde, promotora de equidade, acesso, qualidade e continuidade da atenção ${ }^{13,18,19}$.

Coordenar o cuidado é articular as ações de saúde de forma sincronizada e voltadas ao alcance de um objetivo comum ${ }^{20-23}$, independente do local em que se realizem. É a garantia da continuidade da atenção integrada nos diferentes pontos da rede e compreendida nas dimensões vertical, entre a APS e os demais níveis, e horizontal, na própria equipe de APS, serviços de saúde e equipamentos sociais ${ }^{19,24}$. A coordenação do cuidado possibilita que as intervenções sejam experienciadas pelo usuário de forma contínua, adequadas as suas necessidades e compatíveis com suas expectativas ${ }^{20,23}$; sustenta-se em uma rede de prestadores com canais de comunicação, permitindo que as informações sobre os pacientes sejam transmitidas pelos diversos serviços ${ }^{20,23,24}$. Sua essência é a disponibilidade de informações acerca de problemas anteriores e o reconhecimento de que estas poderão estar relacionadas às necessidades presentes ${ }^{20}$.

No Brasil, o predomínio da APS em territórios vulneráveis e desiguais resulta, em parte, da construção histórica da APS - seletiva -, voltada para controle de doenças específicas em populações pobres ${ }^{25}$, e em parte devido à priorização intencional das políticas públicas em territórios mais vulneráveis, na busca pela equidade do acesso à saúde. Nessas populações a coordenação do cuidado assume uma dimensão de enfrentamento das iniquidades em saúde, constituindo um dispositivo potente de atuação.

Recentemente, no município do Rio de Janeiro (MRJ), se deu uma expressiva expansão da APS. A cobertura passou de 10\% (186 equipes) em maio de 2010, para 64\% (1.251 equipes) em outubro de $2017^{26}$, sendo priorizados os territórios mais vulneráveis para alocação das equipes. Neste contexto, a demanda por atenção especializada tende a aumentar, e a continuidade da atenção integrada às esferas secundária e terciária se torna crucial. Em decorrência, a Secretaria Municipal de Saúde (SMS) fortaleceu o papel da APS na coordenação do cuidado, como estratégia para qualificar o cuidado e o acesso aos serviços especializados, descentralizando a regulação ambulatorial de consultas/exames especializados para as unidades de APS, fortalecendo o vínculo e a coordenação do cuidado neste nível de atenção ${ }^{27}$. Neste período, a coordenação do cuidado pela APS sobressai nas diretrizes da SMS, cujas publicações destacam esse atributo, normatizando fluxos e protocolos de cuidado.

Este trabalho objetivou apresentar a visão de profissionais de saúde a respeito da coordenação do cuidado na esfera da APS e os desafios diante do seu exercício em dois territórios com grandes vulnerabilidades e desigualdades sociais na cidade do Rio de Janeiro.

\section{Método}

Trata-se de pesquisa social em saúde que utilizou o método de Análise de Conteúdo, na modalidade temática, considerada apropriada às investigações qualitativas em saúde ${ }^{28,29}$. Optou-se pela pesquisa social por abranger as investigações que tratam dos problemas sociais, incluindo o fenômeno saúde/doença, e sua representação pelos atores que atuam no campo ${ }^{28,30}$. Foram realizadas entrevistas com profissionais de saúde e observação de campo com utilização de roteiro semiestruturado. As seguintes categorias nortearam a 
análise temática: Significado da coordenação do cuidado para os profissionais de APS; Papel do profissional de APS na coordenação do cuidado e estratégias adotadas; Ferramentas utilizadas na coordenação do cuidado; Dificuldades e desafios na coordenação do cuidado.

O campo de estudo foi constituído por dois territórios que abrangem aglomerados subnormais (favelas) localizados na zona sul do MRJ, na Área Programática (AP) 2.1, considerada modelo em termos de disposição e organização dos serviços de saúde, e com indicadores sociais entre os mais altos do município. Os locais foram definidos em conjunto com gestores da SMS, por apresentarem condições organizacionais e de infraestrutura favoráveis à realização da coordenação do cuidado: equipes de APS completas, unidades de saúde com boa infraestrutura, disposição de materiais e insumos e bom desempenho dos indicadores de processo de trabalho.

O trabalho de campo foi precedido da apresentação do estudo aos gestores do nível central e regional da SMS e do levantamento da composição dos territórios. Foi solicitada indicação de, ao menos, seis unidades de saúde pertencentes a estes territórios, com respectivos contatos de gerentes. Foram escolhidas duas unidades de APS, pela maior receptividade dos gerentes com a proposta da pesquisa. A convite dos gerentes, a escolha das equipes se deu após a apresentação do projeto nas reuniões de unidade, e manifestação de interesse das equipes. Os critérios de inclusão foram quadro de profissionais completo, disponibilidade e interesse de todos integrantes da equipe em participar. Em seguida, a pesquisadora passou a participar das reuniões de equipe; esta inserção aproximou e permitiu o acesso às atividades desenvolvidas nas equipes acompanhadas.

Foram entrevistados os profissionais (oito) das duas equipes de APS: dois médicos, duas enfermeiras, dois técnicos de enfermagem e dois agentes comunitários de saúde (ACS). Apenas um ACS de cada equipe foi entrevistado, indicados pela enfermeira e/ou médico de cada equipe, considerando o comprometimento e envolvimento com as atribuições. Ambos os ACS se disponibilizaram prontamente em participar da pesquisa. Não houve recusa de nenhum profissional para realização das entrevistas. Contudo, houve maior demora no agendamento com os técnicos de enfermagem, sob justificativa de escala de trabalho mais rígida que dificultava sua ausência dos espaços escalados.

A entrada no campo se deu de forma cuidadosa, estabelecendo relação de empatia, respeito e diálogo horizontal, inclusive na escolha conjunta dos espaços e atos a serem observados, não invadindo espaços não autorizados e respeitando os dias combinados para estar presente. As entrevistas foram agendadas com cada profissional conforme sua disponibilidade, realizadas na própria unidade em espaços reservados.

A observação com registro em diário de campo contemplou reuniões de equipe, visitas domiciliares, atividades educativas, consultas de enfermagem e médica, interconsulta com profissionais do Núcleo de Apoio à Saúde da Família (NASF), realização de procedimentos e acolhimento à demanda espontânea; bem como consulta a documentos normativos, publicados pelo município, disponíveis nas unidades ou no formato online. Buscou-se apreender, através do acompanhamento das experiências diárias, nas interações e nas relações mantidas com o campo, o significado e representações que os profissionais atribuíam ao objeto de estudo. A observação participante permitiu uma maior aproximação com o campo e melhor compreensão da complexidade dos processos estudados, funcionando, sobretudo, como complemento às entrevistas.

O trabalho de campo ocorreu entre julho e novembro de 2016. O material de campo foi transcrito e examinado com leitura, exploração e categorização, segundo os núcleos de sentido identificados, descrição e interpretação dos resultados.

O estudo foi aprovado pelo Comitê de Ética em Pesquisa (CEP) do Instituto de Estudos em Saúde Coletiva, da Universidade Federal do Rio de Janeiro e pelo CEP da prefeitura do MRJ.

\section{Resultados}

As equipes investigadas assistem grupos com grande vulnerabilidade social, pertencentes a localidades com alta densidade populacional, moradias irregulares, de difícil acesso, ausência de saneamento e pavimentação e presença de criminalidade e tráfico de drogas. Observou-se alta desigualdade social, evidenciada pela contiguidade com populações de médio e alto poder aquisitivo.

Ambos os territórios são caracterizados por IDH abaixo da média da região. O território 1, com 53.338 habitantes, formado por um conjunto de áreas de aglomerados subnormais, possuía IDH de 0,533 (o menor da região) e renda per capita de até um salário mínimo em $68 \%$ dos domicílios em 2010. O território 2, com população de 21.724 habitantes distribuída parte em áreas de 
aglomerados subnormais e parte em área urbanizada com moradias regulares, possuía, em 2010, IDH de 0,690 e renda per capita média de R\$ 4.149 , sendo que $17 \%$ dos domicílios possuía rendimento domiciliar de até um salário mínimo ${ }^{31}$.

Três unidades de APS estão presentes no território 1 . A unidade de saúde investigada possui 11 equipes de saúde da família e 03 equipes de saúde bucal para atender a uma população adscrita de 30.356 habitantes. A equipe participante do estudo (equipe 1) é composta por 01 médico, 01 enfermeira, 01 técnico de enfermagem e 5 ACS. Segundo informações do prontuário eletrônico, 2.483 pessoas eram acompanhadas pela equipe 1 . Destas, $67 \%$ eram consideradas negras e $65 \%$ beneficiárias de programas de transferências de renda do governo federal e municipal. Aproximadamente $20 \%$ desta população é formada por idosos, considerados os que mais buscam atendimento, juntamente com os portadores de doenças crônicas, gestantes e crianças. Trata-se de população com elevada incidência de tuberculose, registrando em torno de 300 novos casos da doença por 100 mil habitantes a cada ano, reflexo do contexto de pobreza e aglomeração de pessoas ${ }^{32}$.

A unidade de saúde que atende ao território 2 é um centro de saúde formado por 7 equipes de saúde da família, uma equipe de atenção básica com profissionais médicos especialistas e duas equipes de saúde bucal. A equipe participante do estudo (equipe 2), com mesma composição da equipe 1, possui população cadastrada de 2.800 pessoas residentes em aglomerados subnormais na sua totalidade. Em torno de $63 \%$ destas eram negras, $45 \%$ eram beneficiárias dos programas de transferências de renda, e aproximadamente 15\% eram pessoas idosas. Somada às demais populações do território 1 , apresenta a segunda maior taxa de mortalidade infantil $(18,4)$ e neonatal tardia $(9,2)$ da AP $2.1^{31}$.

As duas unidades contavam com programas de residência em Saúde da Família de medicina e enfermagem e profissionais do NASF. Possuem prontuário eletrônico, não integrado às demais unidades de APS e níveis assistenciais. A regulação de vagas ambulatoriais é realizada pelo médico regulador da unidade, através do Sistema de Regulação (SISREG). As equipes dispõem de acesso aos protocolos assistenciais (Guias de Referência Rápida) nos formatos digital e manual, bem como ferramentas de encaminhamento aos serviços especializados (ficha de referência e contrarreferência) no formato impresso. Recebem, via e-mail, relatório de alta hospitalar dos pa- cientes do território ("guia pós-alta hospitalar”) com informações quanto à duração e motivo da internação (Quadro 1).

A rede de saúde, presente na AP 2.1 é composta também por serviços públicos de média e alta complexidade, e serviços da rede suplementar. A contratação/prestação de serviços na APS se dá por contrato com Organizações Sociais em Saúde (OSS), e trabalhadores com contratos regulamentados na Consolidação das Leis do Trabalho (CLT). Este cenário se refere a um período (2009-2016) de investimentos na APS, com expansão, estruturação e qualificação dos serviços.

Os profissionais da APS entendem a coordenação do cuidado, de modo geral, como um processo de comunicação, organização e funcionamento dos serviços em rede, que garante a continuidade e a integralidade da atenção e melhora o acesso e utilização dos serviços. $\mathrm{O}$ entendimento comum da coordenação do cuidado pelas diferentes categorias pode ser atribuído ao destaque dado pelo município ao tema, presente nos documentos normativos que orientam as práticas profissionais e abordado como tema transversal nos espaços de qualificação promovido pela gestão e nas reuniões de equipe. Nota-se pela quantidade de vezes que a frase "coordenação do cuidado" e seus termos correlatos e exemplificações apareceram nos espaços de diálogos acompanhados pela pesquisadora e nos discursos dos entrevistados.

Mencionam especificidades no exercício da coordenação do cuidado pelas diferentes categorias profissionais. As articulações com serviços externos à unidade competem ao médico $\mathrm{e}$ a enfermeira. A dependência da figura do médico para o agendamento de exames, consultas com especialistas e internações foi criticada por restringir a potencialidade da coordenação do cuidado pela APS; assim como a menos-valia atribuída a APS, considerada um empecilho à coordenação. Os técnicos de enfermagem e os ACS participam da coordenação do cuidado no âmbito da unidade, opinando quanto às condutas, aos fluxos e aos encaminhamentos, nos atendimentos compartilhados e reuniões de equipe. Os ACS exercem também papel de articulação com a comunidade, fornecendo informações sobre o contexto de vida e as particularidades das famílias e território, às quais só eles têm acesso, por pertencerem à comunidade e transitarem com maior frequência na casa dos usuários:

Na visita domiciliar, quando observo má condição de higiene, lixo acumulado na porta, água parada, ou, por exemplo, situação de negligência 
Quadro 1. Caracterização dos Sujeitos da Pesquisa.

\begin{tabular}{|l|c|l|l|l|c|}
\hline $\begin{array}{l}\text { Codificação } \\
\text { dos sujeitos }\end{array}$ & Idade & $\begin{array}{l}\text { Sexo } \\
(\mathbf{F} / \mathbf{M})\end{array}$ & Formação Profissional & \multicolumn{1}{|c|}{ Especialização na Área } & $\begin{array}{c}\text { Tempo de Atuação na } \\
\text { APS (anos) }\end{array}$ \\
\hline ACS.Eq1 & 33 & $\mathrm{~F}$ & $\begin{array}{l}\text { Nível médio - Agente de } \\
\text { saúde }\end{array}$ & - & 6 \\
\hline ACS.Eq2 & 49 & $\mathrm{~F}$ & $\begin{array}{l}\text { Nível médio - Agente de } \\
\text { saúde }\end{array}$ & - & $\begin{array}{l}\text { Mestre em Saúde Pública } \\
\text { e Doutoranda em Saúde } \\
\text { Coletiva }\end{array}$ \\
\hline Enf.Eq1 & 32 & $\mathrm{~F}$ & Enfermeira & $\begin{array}{l}\text { Mestrado em Saúde } \\
\text { Coletiva }\end{array}$ & 4 \\
\hline Enf.Eq2 & 37 & $\mathrm{~F}$ & Enfermeira & $\begin{array}{l}\text { Residência de Medicina de } \\
\text { Família e Comunidade }\end{array}$ \\
\hline Med.Eq1 & 34 & $\mathrm{M}$ & Médico & $\begin{array}{l}\text { Residência de Medicina de } \\
\text { Família e Comunidade }\end{array}$ & 3 \\
\hline Med.Eq2 & 27 & $\mathrm{M}$ & Médico & - & 6 \\
\hline Téc.Eq1 & 44 & $\mathrm{~F}$ & Téc. de Enfermagem & - & 4 \\
\hline Téc.Eq2 & 40 & $\mathrm{M}$ & Téc. de Enfermagem & - & \\
\hline
\end{tabular}

Fonte: Elaborado pelas autoras.

no cuidado da criança ou idoso, informo à enfermeira, para ver o que a equipe pode fazer. (ACS. Eq2).

Se estou na visita de um paciente acamado $e$ verifico, com os testes, que ele está com uma possivel úlcera venosa e precisa de um acompanhamento com o angiologista, levo o caso para equipe que discute e faz o processo de encaminhamento no SISREG. (Téc.Eq2).

Há consenso de que profissionais de todos os níveis de atenção devem participar da coordenação do cuidado e, inclusive, o usuário, corresponsável pela própria saúde:

Acho que o papel de coordenação é completamente descentralizado. É meu, do médico, do residente, do ACS, da gerente, do administrativo, da CAP, na função que cabe a cada um. Até o próprio indivíduo é responsável pela coordenação do seu cuidado, autocuidado mesmo. (Enf.Eq1).

A coordenação do cuidado deve ser feita por todos. No nivel da APS, pelos profissionais da equipe e gerente. No nivel da rede, pelos outros serviços de saúde e pela gestão da CAP. (Enf.Eq2).

No entanto, se reconhece, em tese, a primazia da APS como principal ordenadora e coordenadora, constituindo a esfera mais próxima e acessível, para a qual o usuário retorna após $\mathrm{o}$ atendimento especializado, e apropriada para acompanhá-lo em seu percurso nos diversos pontos de atendimento; com maior possibilidade de articular os diferentes níveis de atenção e agregar informações do itinerário terapêutico e do processo de cuidado. A expansão da APS re- quer a coordenação do cuidado para melhorar o acesso aos níveis especializados e sua utilização, especialmente pelos usuários com maiores barreiras de acesso, possibilitando a circulação das pessoas na rede, reduzindo os desfechos negativos e os custos do sistema.

$\mathrm{Na}$ visão dos médicos, coordenar o cuidado implica numa função de filtro, em que a avaliação pela APS antecede o acesso ao especialista. Ao responsável técnico da unidade é atribuída uma dimensão coletiva, política e institucional da coordenação do cuidado, relacionada ao gerenciamento dos fluxos dentro e fora da unidade (fluxos de acesso, acolhimento, referência e encaminhamento). No âmbito da unidade, a coordenação do cuidado acontece mais facilmente, pela proximidade dos serviços e profissionais (equipe de APS, saúde bucal e NASF), facilitando a comunicação entre eles para discussão dos casos compartilhados, bem como o ajuste dos fluxos. A comunicação entre profissionais, destes com os usuários, e com outros níveis de atenção, surge como principal desafio da coordenação do cuidado.

Observou-se que a coordenação do cuidado é praticada diariamente pelos profissionais da APS, embora nem sempre reflitam criticamente sobre essa prática ou tenham consciência dela, pois as ações tendem a se automatizar ao longo do tempo. Mesmo que haja distanciamento da equipe a coordenação ocorre em alguma medida, pois o usuário retorna em busca da continuidade da atenção. 
Com relação às dificuldades, a formação reducionista dos profissionais é vista como fator que inibe a coordenação do cuidado, ao não problematizar o processo saúde-doença e não incentivar a atuação em rede. Essa formação não favorece a compreensão do sistema de saúde como uma rede de serviços, tendo a APS como centro da comunicação, tampouco a percepção da importância da comunicação e coordenação para garantir um cuidado integral e continuado:

Eu acompanhei por um mês o serviço de APS em Londres, na Inglaterra, e lembro que ficava impressionado como era perfeitamente dado e automático que, depois de toda consulta num serviço da atenção secundária, o médico, o enfermeiro, o profissional que tivesse atendido, fazia uma carta de contrarreferência, que não era pequena, para a APS. (Med.Eq2).

A ausência de preenchimento, a insuficiência de informação ou a letra ilegível no formulário de contrarreferência são recorrentes e reforçam a constatação de que a comunicação constitui um entrave:

Incomoda quando a gente encaminha alguém, especifica nossa dúvida na referência, e não recebe a informação do que foi feito no outro serviço. É complicado. A gente não mandou esse paciente pelo prazer de vê-lo se deslocar até o outro lado da cidade ou para se livrar dele, ainda mais quando se trata de grupos vulneráveis como atendemos aqui. A gente encaminhou para receber a contribuição daquele profissional. É complicado quando a informação chega de boca, às vezes o paciente nem sabe dizer o que foi feito ou não sabemos se a informação foi essa mesmo. (Med.Eq2).

A APS gerencia o acesso aos serviços secundários pelo SISREG e a falta de comunicação expressa descompromisso. Cancelamentos por problemas dos serviços não são reagendados e o usuário retorna à APS sem o atendimento. $\mathrm{O}$ campo "notificareg" destina-se ao registro de problemas no agendamento, mas frequentemente o serviço de referência não dá retorno.

As alternativas propostas são a digitação da contrarreferência e a comunicação por e-mail com a equipe de APS, mas o mais lógico seria o SISREG incorporar essas informações. Com relação às internações, o sistema de Alta Referenciada adotado traz o nome do usuário, o motivo e a duração da internação, informações limitadas para coordenar o cuidado. Ocorrem situações de usuários pós-internação sem documento hospitalar informando o diagnóstico e os procedimentos realizados:

Por exemplo, para pacientes terminais, geralmente a gente não fica sabendo oficialmente que não tem mais tratamento curativo e será apenas paliativo em domicílio. Geralmente, a gente vai pensando e chegando a uma conclusão indireta, porque as consultas vão ficando espaçadas, não foi prescrito mais quimioterapia ou ele saiu de alta, mas normalmente a gente não recebe uma carta dizendo que o paciente está fora de possibilidade terapêutica. (Med.Eq1).

A ausência de um sistema integrado de registro em prontuário inibe a troca de informações. Os prontuários eletrônicos não possuem comunicação entre APS e serviços da rede. Integrar os registros de prontuário, permitindo acessar as condutas, resultados de exames e diagnósticos traçados em outros serviços, é um dos grandes desafios identificados pelos profissionais:

O paciente chega 'eu me consultei lá, eles me prescreveram isso'. Mas não tem nada oficial. E não existe nenhum mecanismo de cobrança disso, para que o serviço especializado dê uma resposta à APS. (Med.Eq1).

O compartilhamento de informações e a articulação entre os serviços são muito deficientes. Para as enfermeiras e médicos, essas dificuldades refletem a estrutura fragmentada do sistema e a posição periférica da APS. A APS não é reconhecida pelos serviços especializados como setor responsável pelo cuidado longitudinal e continuado, o coordenador do cuidado. Aliada à fragmentação, a cultura da população, habituada a lógica de consumo de ações e serviços de saúde, contribui para a procura por especialistas sem avaliação prévia da APS.

Os programas de residência médica e multiprofissional; ações de educação permanente; apoio da Coordenadoria de Atenção Primária (CAP); Guias de Referência Rápida, manuais e protocolos instrutivos podem ajudar a reduzir os problemas.

Diante dos avanços pontuais e das dificuldades e desafios enfrentados na coordenação do cuidado pelas equipes, os profissionais advertem para a persistente desvalorização da APS. Há preocupação ante a exclusão da APS da pauta de políticas públicas prioritárias no cenário político brasileiro, especialmente num país onde a maioria dos usuários da APS pertence a populações marginalizadas, pobres e que enfrentam barreiras diversas no acesso e utilização dos serviços de saúde.

\section{Discussão}

Os profissionais entendem a coordenação do cuidado, seu significado, obstáculos e desafios a partir dos respectivos lugares e papéis desempenha- 
dos na equipe e de suas experiências prévias; em consonância ao conceito encontrado na literatu$\mathrm{ra}^{18,20-24}$. Os atributos da $\mathrm{APS}^{15}$ no que tange à integralidade, à continuidade e à longitudinalidade do cuidado, aparecem na visão dos profissionais.

Apesar da preponderância médica, prevalece a compreensão de um papel compartilhado entre os profissionais e com a rede, a ser apreendido pelos profissionais desde a sua formação. Coordenação do cuidado envolve trabalho em equipe, colaboração entre profissionais e integração dos serviços ${ }^{9,24,33,34}$, e também participação dos cidadãos, corresponsáveis pela própria saúde, nos canais participativos de inclusão nos processos decisórios ${ }^{1734-37}$. Vale frisar, a importância do ACS ao integrar informações relativas aos usuários, por meio das visitas domiciliares e busca ativa dos faltosos ${ }^{19}$.

Em alguns estudos a coordenação do cuidado não é prerrogativa da APS, mas atributo organizacional dos serviços de saúde, estratégia do sistema para melhorar a integração entre os níveis assistenciais, traduzida na percepção de continuidade dos cuidados. Entretanto, a APS fortalecida em seus atributos, é reconhecida como ideal para coordenar o percurso terapêutico do usuário, na maioria dos episódios ${ }^{20,23}$. Uma APS forte se caracteriza pela capacidade de resolver a maior parte dos problemas de saúde, em tempo oportuno, com acessibilidade, equidade e continuidade ${ }^{38}$; e ao dispor de apoio, recursos políticos, financeiros e humanos, condiciona a capacidade de coordenar o cuidado ${ }^{14,20,28}$.

Evidencia-se a necessidade de organizar o sistema de saúde com a constituição de redes, nas quais a coordenação do cuidado é assumida como uma responsabilidade ampliada da APS. Mas, apesar das iniciativas de gestores municipais em curso ou implementadas, as dificuldades permanecem, com determinações contextuais que derivam da organização macroestrutural da rede e do apoio governamental ${ }^{17,38,39}$. Neste estudo, as dificuldades identificadas provém das falhas de comunicação entre os serviços, da desvalorização da APS, da baixa qualificação profissional e das fragilidades nos instrumentos adotados para integração dos níveis assistenciais.

A descentralização da regulação é importante para fortalecer a APS como porta de entrada, proporcionar equidade no acesso e aumentar o poder de coordenação do cuidado. Estudo sobre a descentralização da regulação ambulatorial no MRJ, ocorrida em 2012, demonstrou avanço no acesso aos serviços especializados, em virtude do aumento do número de consulta e exames regulados desde então. Sugeriu-se a redução no tempo de espera e aumento da capacidade de coordenação pelas equipes de APS, induzindo maior responsabilidade dos profissionais no acompanhamento dos usuários na realização dos procedimentos regulados por eles ${ }^{40}$. Contudo, este e outros estudos destacam que a insuficiência na oferta de atenção especializada, agravada pela pouca integração entre as redes estadual e federal, gera gargalos artificiais pela ausência de regulação e dificulta o acesso aos serviços especializados $^{17,19,23,40}$.

A ausência de prontuários informatizados integrados e a utilização das tradicionais guias de referência e contrarreferência são apontadas neste e em outros estudos como insuficientes para a organização das RAS com continuidade informacional e coordenação pela APS ${ }^{17,41-43}$.

Estudos sugerem que a coordenação entre níveis assistenciais pode ser facilitada pelo aprimoramento da coleta e disseminação de informações sobre pacientes e prestadores e pela utilização das Tecnologias de Informação e Comunicação, tais como prontuário eletrônico integrado, instrumento informatizado de referência e contrarreferência, ferramentas de regulação, e outras, que possam disponibilizar dados referentes a linha de cuidado dos usuários e permitam a gestão dos processos e fluxos ${ }^{17,21,22,24,38}$. Desse modo, investimento em instrumentos de integração, que permitam acessar condutas, resultados de exames e diagnósticos traçados em diferentes serviços, é fundamental para o gerenciamento do cuidado pela APS. A integração do cuidado depende da qualidade dos processos de coordenação que, por sua vez, dependem da eficácia da comunicação instituída entre as pessoas ${ }^{24}$.

A coordenação do cuidado depende de profissionais qualificados e engajados, cientes da importância de comunicar e partilhar informações. Requer o fortalecimento do trabalho em equipe ${ }^{44}$ e o agir comunicativo pode constituir um caminho para a transformação da prática cotidiana, de uma perspectiva autoritária, fragmentada e individualista para uma visão democrática, integrada, baseada no trabalho coletivo, na solidariedade e na comunicação ${ }^{45}$.

Alguns autores consideram que a APS não reúne condições materiais e simbólicas para exercer o papel de centro comunicador e realizar a conexão entre os vários pontos da rede ${ }^{24,46}$. Logo, o caráter central da APS na constituição das RAS deve se configurar numa relação de mão dupla. Da mesma forma que não existe RAS sem APS robusta capaz de coordenar o cuidado, a APS não 
consegue exercer seu papel sem um sólido arranjo regional e uma articulação virtuosa entre os entes federados ${ }^{47}$.

É fundamental maior investimento em infraestrutura, tecnologia de informação, capacitação de pessoal, fluxos e processos de trabalho; profissionais comprometidos com um processo de trabalho horizontal, em que se combine especialização com interdisciplinaridade; rompimento das características hegemônicas do modelo de atenção atual; fortalecimento do papel do generalista na condução dos cuidados; e usuários e especialistas abertos aos novos modelos organizacionais ${ }^{24}$.

A gestão tem papel primordial, de modo a contribuir para:

[...] um processo de produção em saúde mais eficiente e eficaz em áreas prioritárias e com qualidade, abrindo possibilidade para rearranjos "inteligentes" que se estabeleçam para o enfrentamento ou superação das diversidades e complexidades sociais que se colocam aos serviços de saúde ${ }^{17}$ (p.715).

Países, cujos sistemas de saúde se organizam com base na APS, apresentam melhores níveis de saúde e maior equidade, visto que o acesso e alcance das atividades de projeção comunitária e a oportunidade de atenção continuada e de coordenação do cuidado contribuem para reduzir as iniquidades em saúde ${ }^{4,5,16}$. Logo, os benefícios de uma coordenação do cuidado adequada se traduzem em ganho individual para o usuário e ganho para o sistema, no que tange à alocação de recursos de modo mais equânime entre áreas de maior vulnerabilidade social, auxiliando na redução das desigualdades em saúde ${ }^{20,48}$.
Assim, os desafios para coordenar o cuidado são compartilhados por diversos sistemas de saúde, localidades nacionais e internacionais. Não obstante o contexto desfavorável, é necessário envidar esforços para expor as vantagens dos sistemas baseados na APS, e os benefícios em larga escala para a consolidação dos sistemas de saúde, no que tange à equidade, à acessibilidade, à eficácia clínica e sanitária e à eficiência econômica e que possibilite a integração dos cuidados. Fazse necessário, portanto, estudos como este, que compartilhem experiências locais, apresentando inovações e desafios enfrentados no modo de fazer a coordenação do cuidado na APS, enriquecendo a interlocução e reflexão no campo da saúde coletiva.

Por fim, os limites deste estudo estão na possibilidade dos resultados não refletirem os desafios e as estratégias adotadas em localidades onde a APS não dispõe das ferramentas de coordenação, de profissionais qualificados ou de significativa priorização política, como verificado no período e territórios investigados. A escolha destas localidades, ao considerar também a indicação por parte dos gestores e profissionais levou a um cenário com uma coordenação do cuidado mais efetiva e com maior conscientização e criticidade dos sujeitos a respeito dessa prática. Estudos que investiguem outras localidades, com diferentes perspectivas de análises e maior quantitativo de atores sociais, poderão trazer contrapontos aos resultados obtidos aqui e, assim, ampliar o conhecimento sobre o tema.

\section{Colaboradores}

As autoras contribuíram na concepção, planejamento, análise e interpretação dos dados; escrita, revisão crítica; e versão final do texto. O presente manuscrito deriva da dissertação de mestrado da autora principal, sob orientação da segunda autora. 


\section{Referências}

1. Silva SF. Organização de redes regionalizadas e integradas de atenção à saúde: desafios do Sistema Único de Saúde (Brasil). Cien Saude Colet 2011; 16(6):27532762.

2. Conill EM, Fausto MCR, Giovanella L. Contribuições da análise comparada para um marco abrangente na avaliação de sistemas orientados pela atenção primária na América Latina. Rev Bras Saude Mater Infant 2010; 10(1):14-27.

3. Organização Mundial da Saúde (OMS). Cuidados inovadores para condições crônicas: componentes estruturais de ação. Brasília: OMS; 2003.

4. Neri M, Soares W. Desigualdade social e saúde no Brasil. Cad Saude Publica 2002; 18(Supl):77-87.

5. Organização Pan-Americana da Saúde (OPAS). Módulo de princípios de epidemiologia para o controle de enfermidades. Módulo 1: apresentação e marco conceitual. Brasília: OPAS; 2010.

6. Barreto MS, Carreira L, Marcon SS. Envelhecimento populacional e doenças crônicas: Reflexões sobre os desafios para o Sistema de Saúde Pública. Rev Kairós 2015; 18(1):325-339.

7. Comissão Nacional sobre Determinantes Sociais da Saúde (CNDSS). As causas sociais das iniquidades em saúde no Brasil. Relatório Final da CNDSS [relatório na Internet]. 2008 [acessado 2019 Jan 7]. Disponível em: http://bvsms.saude.gov.br/bvs/publicacoes/causas_sociais_iniquidades.pdf

8. Malta D. No horizonte, o enfrentamento das iniquidades em saúde [entrevista na Internet]. Saúde Amanhã; 2017 [acessado 2019 Fev 20]. Disponível em: https:// saudeamanha.fiocruz.br/no-horizonte-o-enfrentamento-das-iniquidades-em-saude-2/\#.WIjQ-VUrKii

9. Brasil. Portaria no 4.279 , de 30 de dezembro de 2010 . Estabelece diretrizes para a organização da Rede de Atenção à Saúde no âmbito do SUS. Diário Oficial da União 2010; $30 \mathrm{dez}$.

10. Malta DC, Merhy EE. O percurso da linha de cuidado sob a perspectiva das doenças crônicas não transmissíveis. Interface (Botucatu) 2010; 14(34):593-605.

11. Mendes EV. As redes de atenção à saúde. Brasília: OPAS; 2011.

12. Mendes EV. O cuidado das condições crônicas na atenção primária à saúde: o imperativo da consolidação da estratégia da saúde da família. Brasília: OPAS; 2012.

13. Almeida PF, Giovanella L, Nunan BA. Coordenação dos cuidados em saúde pela atenção primária à saúde e suas implicações para a satisfação dos usuários. Saude Debate 2012; 36(94):375-391.

14. Organização Mundial da Saúde (OMS). Atenção Primária à Saúde: agora mais do que nunca. Relatório Mundial de Saúde [relatório na Internet]. Genebra: WHO; 2008 [acessado 2018 Jun 10]. Disponível em: http://189.28.128.100/dab/docs/publicacoes/geral/ rms.pdf

15. Organização Mundial da Saúde (OMS). Informe de la Conferencia Internacional sobre Atención Primaria de Salud [documento na Internet]. Genebra: WHO; 1978 [acessado 2018 Jun 20]. Disponível em: https:// medicinaysociedad.files.wordpress.com/2011/06/declaracion-de-alma-ata.pdf
16. Conselho Nacional de Secretários de Saúde (CONASS). Atenção primária e promoção da saúde [livro na Internet]. Brasília: CONASS; 2011 [acessado 2019 Abr 20]. Disponível em: https://www.conass.org.br/ bibliotecav3/pdfs/colecao2011/livro_3.pdf

17. Lapão LV, Arcêncio RA, Popolin MP, Rodrigues LBB. Atenção Primária à Saúde na coordenação das Redes de Atenção à Saúde no Rio de Janeiro, Brasil, e na região de Lisboa, Portugal. Cien Saude Colet 2017; 22(3):713-723.

18. Martínez DH, Navarrete MLV, Lorenzo IV. Factores que influyen en la coordinación entre niveles asistenciales según la opinión de directivos y profesionales sanitarios. Gac Sanit 2009; 23(4):280-286.

19. Aleluia IRS, Medina MG, Almeida PF, Vilasbôas ALQ. Coordenação do cuidado na atenção primária à saúde: estudo avaliativo em município sede de macrorregião do nordeste brasileiro. Cien Saude Colet 2017; 22(6):1845-1856.

20. Starfield B. Atenção Primária: equilíbrio entre necessidades de saúde, serviços e tecnologia. Brasília: Organização das Nações Unidas para a Educação, a Ciência e a Cultura/MS; 2002.

21. Núñez RT, Lorenzo IV, Naverrete MLV. La coordinación entre niveles asistenciales: uma sistematización de sus instrumentos y medidas. Gac Sanit 2006; 20(6):485-95.

22. Hofmarcher MM, Oxley H, Rusticelli E. Improved Health System Performance Through Better Care Coordination. Paris: OECD; 2007.

23. Almeida PF, Giovanella L, Mendonça MHM, Escorel S. Desafios à coordenação dos cuidados em saúde: estratégias de integração entre níveis assistenciais em grandes centros urbanos. Cad Saude Publica 2010; 26(2):286-298.

24. Rodrigues LBB, Silva PCS, Peruhype RC, Palha PF, Popolin MP, Crispim JÁ, Pinto IC, Monroe AA, Arcêncio RA. A atenção primária à saúde na coordenação das redes de atenção: uma revisão integrativa. Cien Saude Colet 2014; 19(2):343-352.

25. Giovanella L, Mendonça MHM. Atenção Primária à Saúde: seletiva ou coordenadora dos cuidados? [livro na Internet]. Rio de Janeiro: CEBES; 2012 [acessado 2019 Abr 20]. Disponível em: http://cebes.org.br/site/ wp-content/uploads/2015/02/9ATEN\%C3\%87\%C3\%83O-PRIM\%C3\%81RIA-\%C3\%80-SA\%C3\%9ADE.pdf

26. Brasil. Cobertura da Atenção Básica. E-gestor [página na Internet]. 2017. [acessado 2019 Mar 11]. Disponível em: https://egestorab.saude.gov.br/paginas/acessoPublico/relatorios/relHistoricoCoberturaAB.xhtml

27. Rio de Janeiro. Plano Municipal de Saúde do Rio de Janeiro 2014 - 2017. Rio de Janeiro: Secretaria Municipal de Saúde; 2013.

28. Minayo MCS. O Desafio do Conhecimento: pesquisa qualitativa em saúde. 14a ed. São Paulo: Hucitec; 2014.

29. Bardin L. Análise de conteúdo. São Paulo: Edições 70; 2011. 
30. Groulx LH. Contribuição da pesquisa qualitativa à pesquisa social. In. Poupart J, Deslauriers JP, Groulx LH, Laperrière A, Mayer R, Pires AP, Jaccoud M, Cellard A, Houle G, Giorgi A, organizadores. A pesquisa qualitativa: enfoques epistemológicos e metodológicos. 3a ed. Petrópolis: Vozes; 2012. p. 95-126.

31. Rio de Janeiro. DATARIO Informações sobre a Cidade [página na Internet]. [acessado 2019 Nov 11]. Disponível em: http://www.data.rio/search?groupIds=e2fa9e49d6e04512bd9bbbeafb026795\&sort=-modified

32. Rio de Janeiro. Secretaria Municipal de Saúde. TABNET-SUBPAV. Situação de saúde [página na Internet]. [acessado 2019 Nov 11]. Disponível em: https:// subpav.org/tabnet/tabnet_siab_sitsaude_dados.php

33. Organização Pan-Americana de Saúde (OPAS). A atenção à saúde coordenada pela APS: construindo as redes de atenção no SUS. Contribuições para o debate. Brasília: OPAS; 2011.

34. Brown R, Schore J, Archibald N, Chen A, Peikes D, Sautter K, Lavin B, Aliotta S, Ensor T. Coordinating Care for Medicare Beneficiaries: Early Experiences of Demonstration Programs, Their Patients, and Providers. Princeton: Mathematica Policy Research; 2004.

35. Vitor AF, Lopes MVO, Araujo TL. Teoria do Déficit de Autocuidado: Análise da sua Importância e Aplicabilidade a Prática de Enfermagem. Esc Anna Nery 2010; 3(14):611-616.

36. Weenink JW1, van Lieshout J, Jung HP, Wensing M. Patient Care Teams in treatment of diabetes and chronic heart failure in primary care: an observational networks study. Implement Sci 2011; 6:66.

37. Silva RM, Andrade LOM. Coordenação dos cuidados em saúde no Brasil: o desafio federal de fortalecer a atenção primária à saúde. Physis Rev Saude Colet 2014; 24(4):1207-1228.

38. Feo JO, Campo JMF, Camacho JG. La coordinación entre Atención Primaria y Especializada: reforma del sistema sanitario o reforma del ejercicio professional. Rev Adm Sanit 2006; 4(2):357-382.

39. Almeida PF, Fausto MCR, Giovanella L. Fortalecimento da atenção primária à saúde: estratégia para potencializar a coordenação dos cuidados. Rev Panam Salud Publica 2011; 29(2):84-95.

40. Pinto LF, Soranz D, Scardua MT, Silva IM. A regulação municipal ambulatorial de serviços do SUS no Rio de Janeiro: avanços, limites e desafios. Cien Saude Colet 2017; 22(4):1257-1267.

41. Chan B, Proudfoot J, Zwar N, Davies GP, Harris MF. Satisfaction with referral relationships between general practice and allied Health professionals in Australian Primary Health care. Aust J Prim Health 2011; $17(3): 250-258$.
42. Schang L, Waibel S, Thomson S. Measuring care coordination: health system and patient perspectives. London: LSE Health; 2013.

43. Almeida PF, Santos AM. Diálogos em busca de coordenação do cuidado: linha de chegada ou novo itinerário? In: Almeida PF, Santos AM, organizadores. Atenção Primária à Saúde na coordenação do cuidado em Regiões de Saúde. Salvador: EDUFBA; 2015. p. $277-$ 303.

44. Souza MB, Rocha PM, Sá AB, Uchoa SAC. Trabalho em equipe na atenção primária: a experiência de Portugal. Rev Panam Salud Publica 2013; 33(3):190-195.

45. Lima JC, Rivera FJU. Agir comunicativo, redes de conversação e coordenação em serviços de saúde: uma perspectiva teórico-metodológica. Interface (Botucatu) 2009; 12(31):329-342.

46. Cecílio LCO, Andreazza R, Carapinheiro G, Araújo EC, Oliveira LA, Andrade MGG, Meneses CS, Pinto NRS, Reis DO, Santiago S, Souza ALM, Spedo SM. A Atenção Básica à Saúde e a construção das redes temáticas de saúde: qual pode ser o seu papel? Cien Saude Colet 2012; 17(11):2893-2902.

47. Bousquat A, Giovanella L, Campos EMS, Almeida PF, Martins CL, Mota PHS, Mendonça MHM, Medina MG, Viana ALA, Fausto MCR, Paula DB. Atenção primária à saúde e coordenação do cuidado nas regiões de saúde: perspectiva de gestores e usuários. Cien Saude Colet 2017; 22(4):1141-1154.

48. Soranz D, Pinto LF, Camacho LAB. Análise dos atributos dos cuidados primários em saúde utilizando os prontuários eletrônicos na cidade do Rio de Janeiro. Cien Saude Colet 2017; 22(3):819-830.

Artigo apresentado em 30/05/2019

Aprovado em 07/08/2019

Versão final apresentada em 22/11/2019 